\title{
IMPORTANCIA DE LOS HERBARIOS EN EL MANEJO Y APROVECHAMIENTO DE LOS BOSQUES
}

\author{
Reyes Bonilla Beas* \\ Luciano Vela Gáldez * *
}

\section{INTRODUCCION}

México, por sus características topográficas, cuenta con una amplia gama de condiciones ecológicas lo que a su vez se traduce en una gran variedad de su flora y tipos de vegetación de los cuales, Miranda y Hernández X, distinguen 32 en todo el territorio Nacional, entre los que se encuentran los pinares, bosques de escuamifolios y los encinares de las zonas templadas, varios tipos de selva de las zonas tropicales a la vez que, otros tipos de las zonas áridas de los que forman parte especies que son de gran utilidad para el hombre por los productos que de ellas obtiene.

El aprovechamiento de estos recursos presenta varios aspectos los que se pueden agrupar en tres grandes líneas generales que son:

La formación profesional de los técnicos que tendrán bajo su responsabilidad, la dirección de todos los trabajos dirigidos al aprovechamiento de los recursos forestales.

La realización práctica de estos aprovechamientos mediante la aplicación de técnicas modemas que permitan un rendimiento sostenido sin poner en peligro la existencia misma del recurso.

Los trabajos de investigación dirigidos a la búsqueda de nuevas técnicas que permitan un aprovechamiento más adecuado de los recursos mediante un conocimiento más profundo de los mismos sobre su cuantía y ubicación dentro del territorio nacional, dando a los productos del bosque el uso más conveniente y aplicando métodos de control y combate de plagas y enfermedades más efectivos.

Dentro de este enfoque del problema, los herbarios juegan un papel muy importante, desgraciadamente no perceptible muy fácilmente por lo que, es

* Escuela Nacional de Agricultura, Chapingo, Méx.

** Instituto Nacional de Investigaciones Forestales. Coyoacán, D. F. 
necesario explicar con claridad en donde reside esa importancia, que de ninguna manera es pequeña sino que, por el contrario, forma parte muy destacada dentro del conjunto de instrumentos de los cuales hay que echar mano en el aprovechamiento correcto de los bosques.

Desgraciadamente y, no obstante la riqueza florística de nuestro país y a pesar del esfuerzo que realizan los centros de enseñanza Superior así como otras instituciones de carácter científico, el incremento y uso de los herbarios no ha merecido toda la atención que su importancia reclama de tal manera que en la actualidad, dentro del área metropolitana y lugares circunvecinos, solamente se cuentan con los herbarios siguientes: el de la Universidad Nacional Autónoma de México; el de la Escuela Nacional de Ciencias Biológicas del Instituto Politécnico Nacional y el del Instituto Nacional de Investigaciones Forestales. Además, se encuentran en formación, los herbarios de la Escuela Nacional de Agricultura, el de la Comisión para el Estudio del Territorio Nacional y el del Instituto Nacional de Investigaciones Agrícolas.

Los herbarios de la Universidad Nacional Autónoma de México, el de la Escuela Nacional de Ciencias Biológicas y el de la Escuela Nacional de Agricultura de Chapingo (en formación), se orientan fundamentalmente a la enseñanza mientras que el del Instituto Nacional de Investigaciones Forestales tiene como objetivo principal, servir de apoyo a los trabajos de investigación.

\section{PAPEL DE LOS HERBARIOS EN EL MANEJO Y APROVECHAMIENTO DE LOS BOSQUES}

Los recursos forestales constituyen una de las riquezas más importantes con que cuenta México. De acuerdo con la información proporcionada por el Inventario Nacional Forestal, la superficie arbolada del país se estima en 4,0,985,106 ha., de las cuales $29,587,484$ ha., son de clima templado y frío y 11,397,622 ha., se encuentran ubicadas en regiones de clima tropical y subtropical (Cámara Nacional de la Silvicultura, 1970).

Los bosques de clima templado y frío se encuentran en las partes altas de las montañas y por su contribución a la producción forestal total del país, son los que revisten mayor importancia ya que, de acuerdo con los datos estadísticos de la Subsecretaría Forestal y de la Fauna, los bosques de coníferas, estimados en 14,799,578 ha., contribuyen con más del 70\% del valor total de la producción; además, deben considerarse 3,401,715 ha., de latifoliadas, entre las que, los encinos, están cobrando importancia creciente con bastante rapidez; 
estas masas forestales, por su cantidad y facilidad de manejo, son la base para el establecimiento de una amplia gama de industrias forestales.

El aprovechamiento racional u ordenación de las comunidades forestales de clima templado y tropical, es diferente. Si bien es cierto que en las zonas templadas y frías, los bosques de coníferas no representan problemas muy difíciles, debido a la simplicidad en cuanto al número de especies que los constituyen, en esta gran superficie prosperan una serie de especies, variedades y formas que todavía se encuentran imperfectamente estudiadas y que por lo mismo, requieren de más estudios botánicos y ecológicos.

Como ejemplo se puede mencionar la familia de las Fagaceae, de la cual se calcula que existen, dentro de nuestro territorio, 280 especies del género Quercus (1964, Botánica Forestal) y que, debido a problemas taxonómicos y la falta de muestras botánicas en los herbarios existentes en el país, se tienen serios problemas para dar a conocer a los estudiantes sus características e información para el correcto manejo y explotación de este grupo tan importante en la flora mexicana.

En las zonas tropicales el problema es más difícil por la mezcla de especies que se presentan formando parte de las masas forestales.

Es en éste punto donde intervienen los conocimientos botánicos ya que los técnicos encargados del aprovechamiento deben tener amplios conocimientos sobre la materia, pues dado a que cada especie tiene sus propios requerimientos ecológicos, la calidad de sus productos es específica y en muchos casos existe una relación muy estrecha entre huéspedes y parásitos.

Además, es necesario al hacer el estudio florístico y ecológico de las masas forestales, tomar en consideración a toda la vegetación: árboles, arbustos y hierbas, ya que el bosque, como tal, actúa como una unidad en la que, cada uno de sus constituyentes, tiene una función que desempeñar, importante para todo el conjunto.

Los conocimientos botánicos son indispensables en los tres niveles que se han mencionado con anterioridad. En la enseñanza forestal son necesarios puesto que las instituciones encargadas de preparar a los profesionistas forestales deben darles los elementos básicos para el desempeño de su cometido y entre dichos elementos está el conocimiento de las especies constituyentes de los bosques.

Una forma de llegar a este conocimiento sería la de organizar viajes a todas las áreas boscosas para que el estudiante conociera en su medio y en vivo, todas las especies con las que habrá de trabajar en el desempeño de su ejercicio profesional. Sin embargo, este procedimiento alargaría demasiado el tiempo de estudio además de que, elevaría sensiblemente cl costo de la enseñan- 
za, razón por la cual, en este aspecto, los centros de enseñanza tienen que restringirse a pocos viajes de exploración, a lugares seleccionados con anterioridad, pero que de ninguna manera permiten al estudiante conocer todas las especies forestales que existen, ni todas las variaciones que presenta cada una de las especies; es decir, la información que se obtiene es, por necesidad muy restringida.

Otra alternativa que se presenta, la más práctica a fin de cuentas, es el empleo de los herbarios.

Ciertamente en un herbario solamente es posible observar una parte de la planta y además, fuera de su habitat natural. A veces es posible contar con hojas, flores y frutos pero muy frecuentemente, sólo se dispone de las hojas, y tratándose de árboles, jamás se podrá tener a la vista el vegetal completo. En estas condiciones únicamente se puede adquirir un conocimiento parcial.

Esta falla natural, obligada, de los herbarios, se subsana en buena parte con las anotaciones que el colector hace en la etiqueta que se adhiere a la cartulina donde se monta el ejemplar antes de ser herborizado.

Un colector experimentado es capaz de describir en pocas palabras, las características fundamentales de la planta y del medio en que se desarrolla a grado tal que, quien recoge esa información no tendrá gran dificultad en reconocerla en el campo y además con relativa facilidad puede llegar al lugar donde se encuentra.

Por otra parte, es sabido que el tamaño de los individuos de una población así como los integrantes de todas las poblaciones es variable, así como las características de sus órganos: hojas, flores y frutos, de tal manera que cuando tenemos a la vista un individuo o una muestra de él, no tenemos la seguridad de que sus características representan los valores más comunes dentro de la especie.

En este aspecto, los herbarios muestran una superioridad con respecto a otros medios de conocimiento puesto que rápidamente y en el mismo lugar el estudiante puede captar, entre más completas sean las colecciones mejor, la gama de variación que se presenta en toda el área de distribución de las especies, variación no sólo en el aspecto morfológico sino también en cuanto al habitat en que se desarrollan las diversas poblaciones de la misma especie.

Consideramos que cualquier estudiante que se prepare para actuar dentro del marco del aprovechamiento forestal, debe tener en el herbario, una de sus más importantes fuentes de preparación; al mismo tiempo y como parte indispensable de lo anterior, los herbarios deben ampliarse a fin de que cuenten 
con colecciones lo más completas posibles que representen todas las variaciones morfológicas y ecológicas de cada especie.

Sin embargo, por muy amplia que sea la preparación botánica que reciba el estudiante, éste al terminar sus estudios no podrá estar en condiciones de conocer todas las especies forestales susceptibles de aprovechamiento y por lo mismo durante su ejercicio profesional se verá forzado a seguir ampliando sus conocimientos sobre la materia.

Varios son los factores que le obligan a ello. Por una parte, al ordenar sus bosques forzosamente debe tomar en consideración las características, en cuanto a su crecimiento, de cada una de las especies constituyentes de la masa forestal que está manejando y es lógico que, para poder hacer esto, debe partir de lo más elemental: conocerlas y saber distinguirlas en el campo; esto requiere de conocimientos botánicos lo más amplio posibles.

Por otra parte la industria que usa como materia prima la madera u otros productos del bosque, cada día es más exigente en cuanto a sus requerimientos, lo que obliga a quienes hacen el aprovechamiento primario del bosque, a seleccionar las especies más adecuadas a los fines que se persiguen.

Esto nos conduce nuevamente al mismo punto que señalamos en el caso anterior, la necesidad de conocer perfectamente las especies con que se está trabajando.

Está claro que el profesionista forestal puede echar mano de las descripciones de especies que existen en las floras, para poder identificar las que le son desconocidas, pero una identificación de esta manera siempre será incierta si no se comparan las muestras de las plantas en estudio con material debidamente identificado y este material, lo encontramos en los herbarios.

Por consiguiente, cualquier profesionista ligado de una u otra manera con el aprovechamiento de los bosques debe estar familiarizado con el uso de los herbarios a fin de que pueda estar en condiciones de resolver cualquier duda que se le presente en el desempeño de su actividad, por lo que respecta al conocimiento de las especies que está manejando.

De otra manera, si no se conocen las especies que se están aprovechando no será posible hacer aportaciones importantes al desarrollo de las industrias derivadas de la silvicultura y sí, por el contrario, se está sirviendo de obstáculo, en cierta medida, para dicho desarrollo.

Pasando a otro campo, podemos afirmar que la investigación forestal es fundamental en el correcto aprevechamiento de nuestros recursos bo cosos. No solamente existe la necesidad de aumentar el volumen de productos forestales que se pongan a disposición del público consumidor, sino que es necesario 
dar a cada uno de ellos el uso más adecuado de tal manera que su valor aumente y además también se requiere encontrar sustitutos, dentro de los elementos de la flora mexicana, para aquellos productos de importación.

Un planteamiento de esta naturaleza, conduce a la investigación forestal a llevar a cabo estudios tecnológicos muy cuidadosos de los productos del bosque, ya se trate de madera, resinas a cualquier otro producto y decidir qué especie cumple mejor con los requerimientos exigidos para un fin determinado; necesita conocer lo mejor posible, los constituyentes de la flora mexicana para poder dar a las empresas de cualquier tipo, que se dedican al aprovechamiento de los bosques, una evaluación correcta de recursos disponibles, su localización y cuantía, a fin de que las inversiones se planifiquen de la mejor manera; requicre conocer los aspectos básicos de la ecología de las especies aprovechables a fin de que pueda organizarse su ordenación y manejo; requiere encontrar métodos prácticos de cultivo para impulsar la ampliación de las áreas ocupadas por especies valiosas, ya sean nativas o introducidas y requiere, finalmente de trabajos de mejoramiento genético que conduzca a obtener productos de mejor calidad.

Desde el punto de vista silvícola, relacionado con la Enseñanza y la Investigación, nos interesan no sólo los métodos de cultivo y tratamientos que se siguen en el bosque, para su protección y fomento, sino que el buen manejo del bosque se inicia al defenderlo de todos los agentes destructivos: plagas, enfermedades, incendios, pastoreo, desmontes y explotaciones irracionales, buscando que las especies que prosperan en las masas forestales aseguren su regeneración, y que éstas al irse desarrollando proporcionen los árboles que al llegar a la edad de corta deben aprovecharse como cosecha natural del bosque.

Para esto debemos tener un conocimiento de todas las especies vegetales; es decir, qué características presentan en cuanto a su crecimiento, en qué condiciones ecológicas prosperan, datos importantes para la introducción de especies de alto rendimiento con el fin de obtener una mayor productividad por unidad de superficie.

También son interesantes desde el punto de vista de la Investigación los as. pectos fenológicos tales como la época de floración y fructificación de las plantas, información útil en la tarea de obtención de semilla de las especies forestales nativas con el propósito de surtir la demanda tanto interna como la que proviene del extranjero, para lo cual se debe tener mucho cuidado en que la semilla que se pone a disposición del mercado corresponda a la especie que solicita el comprador, ya que de otra manera se cometerían errores muy costosos en cuanto a inversión de tiempo, capital y esfuerzo. 
Además de la recolección y venta de semillas, las investigaciones de mejoramiento se orientan hacia el aspecto genético con el propósito de encontrar variedades, híbridos o eco-tipos de mayor rendimiento no sólo en cuanto a volumen de madera producida, sino también en lo que se refiere a la calidad de las mismas y para estos estudios debe partirse de la identificación más cuidadosa que sea posible de las especies con las cuáles se trabaja, ya que de otra manera los resultados de la investigación perderían en gran parte su valor.

Referente a los factores destructores de las masas forestales, el control de las plagas y enfermedades debe estar precedido de la correcta identificación de las especies tanto huéspedes como parásitos ya que, es bien conocida la relación que existe, desde el punto de vista ecológico, entre los dos niveles de la asociación.

En cuanto a los problemas de pastoreo en los bosques, como no existe un estudio sobre las gramíneas y otras plantas que utilizan los animales, el pastor lo que hace es quemar y con esto destruye el repoblado, daña a los árboles grandes, provocando debilitamiento, lo que da origen a plagas y enfermedades que a la postre destruyen las masas forestales; conociendo las plantas forrajeras que prosperan en los bosques, se podría incrementar su abundancia en áreas apropiadas, evitando de esta manera daños causados a través de los incendios y establecer un pastoreo racional en beneficio de los moradores del campo.

Sobre la política de desmontes que está siguiendo la SAG para la agricultura y ganadería, cabe lamentarse que éstos se hacen, con frecuencia, con desconocimiento de las especies maderables que prosperan en esas áreas; estos desmontes y la tala inmoderada se hace muchas veces sin conocimiento de la dinámica del bosque y sin una evaluación previa de la productividad del suelo bajo diferentes usos. Si bien es cierto que se tiene demanda de productos agrícolas, los desmontes deberían ser precedidos del estudio de todas las plantas de esas áreas para buscar su utilización y no conviene quemarlas como se hace actual. mente en muchas áreas tropicales, es decir las plantas se deben estudiar tanto desde el punto de vista botánico sistemático, como también de la determinación de las características anatómicas, histológicas y las propiedades físicas, químicas y mecánicas de la madera de éstos para buscar un aprovechamiento racional de nuestros recursos vegetales.

Con todo lo dicho hasta aquí, no se requiere de mucha perspicacia para darse cuenta que en la base de todas las investigaciones en el campo forestal están los estudios botánicos, ya que antes de iniciar cualquier investigación, debe procederse a la correcta identificación botánica de la especie o especies 
por estudiar, pues de otra manera, la investigación por muy buena que sea carecerá de todo valor.

Ello es así porque antes que todo, cualquier investigación debe estar referida a una especie determinada, pues de otro modo perdería todo su valor. Unos pocos ejemplos ayudarán a comprender lo anterior.

Caballero Deloya (1968) hizo una evaluación del crecimiento de 28 especies de pino que viven en Pinetum del INIF (21 especies mexicanas y 7 extranjeras). De su estudio queda de manifiesto que bajo las condiciones ecológicas del citado Pinetum, de las especies mexicanas estudiadas, Pinus herrerai y $P$. patula son las que han dado mejores resultados.

Si no se hubiese conocido las especies involucradas en la investigación, lo más que hubiese podido decirse era que, de todas ellas dos eran las más prometedoras y cuando mucho podría haberse dado una descripción de los individuos, pero nada más. Esta información difícilmente pudiera ser utilizable en plantaciones.

Por el contrario, al dar los nombres específicos, cualquier silvicultor que desee aprovechar la información producida en el estudio que se comenta, sobe con precisión el material que necesita así como dónde puede obtener semilla o plántula.

Hernández Sánchez y Salinas Q., al someter a diferentes intensidades de riego plántulas de 6 especies mexicanas de pinos, encontraron que dos de ellas alcanzan su óptimo desarrollo con riegos cada 4-6 días mientras que 4 lo legran con riegos cada 6-9 días.

En este caso, como en el precedente, no todas las especies tienen las mismas exigencias en cuanto a humedad; sin embargo, como las especies a las cuales pertenecían las poblaciones analizadas, estaban perfectamente identificadas, no existe dificultad para aprovechar el resultado de la experiencia.

La demanda de madera de encino va en aumento cada día, sin embargo, sabemos que no todas las especies son utilizables, ya que en algunas las poblaciones están constituidas por individuos de porte bajo, en otras se hallan restringidas a áreas muy reducidas o bien en regiones poco accesibles por el momento. Por lo tanto, el adecuado aprovechamiento de esta riqueza está condicionado, en buena medida, a estudios botánicos que permitan conocer con la mayor precisión posible, las especies con que cuenta la flora mexicana; las características morfológicas de cada una de ellas, principalmente altura y diámetro promedios, rectitud del fuste comercial, características tecnológicas de sus maderas y contenido de taninos de la corteza, su distribución y abundancia dentro del territorio nacional, velocidad de crecimiento, requerimientos ecológicos, etc. 
En otras palabras, está haciendo falta una intensificación de los estudios botánicos para poder proporcionar a la industria de la madera, la información que está requiriendo con demasiada urgencia.

Resumiendo todo lo dicho hasta ahora, creemos haber demostrado que todas las actividades relacionadas con el aprovechamiento de los bosques están estrechamente ligadas a los estudios botánicos. Estos deben preceder a los estudios ecológicos de las especies comercialmente importantes, a las investigaciones tecnológicas sobre calidad de los productos del bosque, a los esfuerzos para mejorar la calidad genética de poblaciones pertenecientes a las especies forestales con que se cuenta, a los trabajos silvícolas y de ordenación de los bosques bajo aprovechamiento y además, como consecuencia de todo esto, deben formar parte importante en la preparación de los profesionistas forestales.

Esta necesidad de los estudios botánicos es lo que determina la importancia de los herbarios ya que, como se ha señalado anteriormente, son una herramienta imprescindible en esta clase de estudios. Por lo mismo, entre mayor sea el número de especies y de áreas geográficas representadas en ellos y entre más amplias sean sus colecciones, el servicio que prestan será de mejor calidad.

Asimismo, queda de manifiesto la necesidad de que tanto estudiantes, profesionistas encuadrados dentro de la actividad forestal e investigadores en este campo, vean en los herbarios uno de sus instrumentos de trabajo más valiosos.

\section{PROBLEMAS :}

No obstante el esfuerzo que están haciendo algunas instituciones de enseñanza superior y de investigación para fomentar la formación y ampliación de los herbarios, aún nos falta un largo camino por recorrer. Varios factores afectan de manera significativa este esfuerzo y de los cuales mencionaremos algunos:

a.--Falta cabal comprensión del significado práctico que tienen los herbarios, lo que se traduce en la falta de apoyo económico amplio y suficiente.

b.-Debido a ello los investigadores que se dedican a la colección, identificación y herborización de material botánico con frecuencia carecen del estímulo económico necesario que les haga posible rendir mejores frutos.

c.-En la mayoría de los herbarios que se dedican a la Enseñanza, se carece de medios materiales, muchas veces los más indispensables y de personal debidamente entrenado.

d.-Falta coordinación entre las instituciones para establecer un sistema de 
intercambio de material lo que permitiría un crecimiento más rápido de los herbarios, con una reducción sensible de costos y de esfuerzo.

e.-Los programas de clases y asignaturas que imparten las Instituciones de Enseñanza Superior no están estructurados de tal manera que se motive al estudiante para crear en él un espíritu de investigación y muchas de las materias básicas necesarias en las carreras se ofrecen como optativas.

f.-Existe un desconocimiento casi total de la importancia que representa el uso de los herbarios en el aprovechamiento forestal, además de que son el archivo para el desarrollo de una serie de estudios científicos (Ecología, Fitogeografía, Etnobotánica, Paleobotánica, Sistemática, Palinología, etc.); esto conduce a que las inversiones de tiempo y dinero, hechas en la formación y ampliación de los herbarios se desaprovechan en gran parte.

g.-A veces, el profesionista que se dedica a estudios botánicos, lo hace en forma rutinaria, con bajo rendimiento de su actividad, debido a que no está debidamente entrenado para llevar a cabo esta actividad o le falta motivación que le haga darse cuenta de la importancia que para el país tiene el esfuerzo que está desarrollando. Quien se dedica a estas actividades está contribuyendo al bienestar humano que debe ser el fin último de toda actividad del hombre.

h.-El Gobierno Federal y los Estatales hasta hace poco habían mostrado muy poco interés en el correcto aprovechamiento e incremento de nuestros recursos naturales, lo que se reflejaba en el desarrollo de los herbarios.

i.-En la enseñanza de las Ciencias Biológicas existe también una desvinculación respecto a la realidad en la serie de problemas socio-económicos que se tienen en el campo; aquí hace falta un nuevo planteamiento respecto a la serie de asignaturas que se imparten en las Escuelas Superiores a nivel de licenciatura; debe haber un balance en las materias aplicadas y aquéllas de tipo básico para poder interpretar e investigar los problemas que se les presentan y darle a esa investigación el uso correcto.

j.-Otro punto muy importante es que para una serie de asignaturas rela. cionadas con la Biología no se cuenta con el material necesario para el gran número de prácticas que se deben tener como complemento de las mismas. Los herbarios bien instalados son un auxiliar insubstituible para la serie de cursos de Botánica que se imparten. Consideramos de gran importancia que cada Institución de Enseñanza debe buscar tener un herbario bien instalado y a la vez planear lo mejor posible las prácticas de campo en donde los alumnos junto con sus profesores conozcan el medio ecológico y la serie de problemas de tipo social que se tienen en el agro mexicano.

k.-Se requiere la existencia de un organismo que de a conocer todos los 


\section{HERBARIOS Y USOS DE BOSQUES}

estudios que se hagan sobre la evaluación de los Recursos Naturales. Hasta ahora son muy pocas las instituciones en donde se han establecido intercambios de material botánico para estudios técnicos y científicos a nivel institucional, y este intercambio cuando menos sería deseable en aquellas áreas que tienen más relación entre sí.

1.-Es indudable que los egresados en las Ciencias Biológicas (Biólogos, Agrónomos, Ingenieros Agrónomos, Zóologos), una vez que salen a prestar sus servicios los absorbe el trabajo, pero si se hace conciencia, cada uno de ellos podría contribuir al fortalecimiento de los herbarios, enviando material a sus respectivas Instituciones; plantas vivas, muertas, semillas, frutos, todas estas serían aportaciones muy valiosas para los herbarios en donde se tendría un conocimiento más amplio de las especies, respecto a su localización, usos y ecología.

m.-A nivel institucional, se cuenta con un gran número de egresados que trabajan en diferentes partes del país, encuadrados dentro de la actividad forestal. Se podría solicitar a las Agencias de Agricultura u otras instituciones, que enviaran material botánico para fortalecer los herbarios: esto sería de gran importancia y ahorraría esfuerzo y dinero al país, puesto que no se tendría que mandar personal a colectar el material a lugares lejanos; claro está que esto requiere de un programa bien meditado en cuanto a su ejecución, además de contar con instructivos precisos sobre las características que deben reunir las muestras que se colectan.

Estos son algunos de los problemas que contribuyen al lento desarrollo de los herbarios del país, pero ellos no son insolubles y debe programarse una actividad dirigida a eliminarlos o reducirlos al mínimo posible a fin de que estos instrumentos de trabajo tan valiosos como son los herbarios se fortalezcan en la medida de lo posible.

\section{CONCLUSIONES :}

De todo lo que se ha dicho hasta este momento, podemos llegar a ciertas conclusiones que nos parecen válidas y que son:

Primera.-Los herbarios son instrumentos básicos de trabajo en toda la actividad forestal. Por lo tanto, todas aquellas instituciones y organismos, públicos o privados, que de una manera u otra, estén ligados con el aprovechamiento de los recursos naturales renovables, deben interesarse en ayudar al estableci- 
miento, ampliación y conservación de los herbarios principalmente aquellos que se ligan a la actividad forestal como son el del Instituto Nacional de Investigaciones Forestales, Escuela Nacional de Agricultura de Chapingo, Escuela Nacional de Ciencias Biológicas, Instituto de Biología de la UNAM y de otras instituciones de enseñanza donde se preparan profesionistas que irán a laborar en las actividades relacionadas con el aprovechamiento de los bosques.

Segunda.-Deben buscarse mecanismos que permitan establecer un intercambio expedito de material botánico a fin de ampliar los herbarios existentes con el menor costo posible.

Tercera.--Los colectores botánicos, autorizados por la Subsecretaría Forestal y de la Fauna, deben cumplir con la obligación que se les impone, de enviar duplicados de sus colectas al INIF y la Universidad y hacer extensiva esta obligación para con la Escuela Nacional de Agricultura y la de Ciencias Biológicas del IPN.

Cuarta.-Debe estimularse económicamente a los profesionales de la botánica a fin de contar con un mayor número de ellos, dada la urgencia que de los mismos tiene el país.

Quinta.-Debe motivarse y capacitarse al personal que labora en los herbarios a fin de que, su actividad sea más fructífera en favor de una mejor calidad de los servicios prestados por los mismos.

Sexta.--Todos los profesionistas cuya actividad está ligada con el aprovechamiento de los recursos forestales, deben considerar el uso de los herbarios como uno de sus instrumentos de trabajo más importantes.

Séptima.-Es necesario promover el apoyo oficial y privado para los herbarios, dado el papel que desempeñan en el correcto aprovechamiento de los recursos forestales y por lo mismo, forman parte destacada de los programas de desarrollo de las comunidades rurales. 